\title{
Report of the Third Workshop of the EASD Study Group on Artificial Insulin Delivery Systems, Pancreas and Islet Transplantation (AIDSPIT)
}

\author{
5-7 February 1984, Igls, Austria
}

\author{
E.F.Pfeiffer, Chairman of the Study Group
}

During the first part of the Workshop, pancreas transplantation, islet transplantation and immunosuppression were discussed. Dr. C.-G.Groth (Stockholm) gave a brief report on the present state of the art of pancreatic (segmental) transplantation. Until June 1983 a total of 280 transplantations had been performed. In July 1983, 57 of these transplants were functioning (22\%), $11 \%$ for more than 12 months. During the last 5 years a steady increase in the number of segmental transplants has been observed. In most cases simultaneous transplantation of pancreas and kidney was performed (144 cases); in 65 cases the pancreas was transplanted after kidney grafting, and in 64 cases the pancreas transplantation was done alone. The longest duration of a living recipient with a functioning pancreas was $5 \frac{1 / 2}{2}$ years. Compared with 1981 and 1983, no further improvement in the percentage of functioning grafts was seen.

Dr. J.Traeger (Lyon) reported on the results of the Lyon-Milan group. The use of cyclosporin A instead of azathioprine was accompanied by an improvement in the results of intravenous glucose tolerance tests. Loss of pancreatic grafts was due to: rejection in $18 \%$ of patients, thrombosis in $12 \%$, death in $33 \%$, hepatitis in $1 \%$, other reasons in $36 \%$. According to his studies, no marker for rejection of pancreatic tissue is at present available. There was now recurrence of insulitis in his pancreatic grafts.

During the discussion, Dr. D.E.R. Sutherland's observation of insulitis within segmental grafts of identical twins was mentioned. No European group has made similar observations.

Dr. R. Landgraf (Munich) reported on 21 patients with simultaneous grafting of pancreatic segments and kidney. At present $71 \%$ of the pancreatic grafts were functioning. Comparison between the data of the Munich group and others is difficult because of the different periods of observation. Dr. Landgraf reported one patient with improvement of retinopathy after pancreas transplantation.

Dr. G. Tyden (Stockholm) studied the influence of cyclosporin A in patients with pancreatic grafts. He observed elevation of creatinine levels, and a deterioration in the intravenous glucose tolerance test even though C-peptide levels increased. Cortisol levels also increased during cyclosporin A treatment. According to Dr. C. R. Stiller (Ontario) cyclosporin A leads to a competitive inhibition of liver enzymes, thus explaining the high cortisol levels.

Dr. P. Lacy (St. Louis) gave a detailed description of new developments in experimental islet transplantation. His attempts to induce tolerance of allogeneic islets were very successful. Using the following techniques, allogeneic islets transplanted across a major histocompatibility barrier survived 100 or even 200 days: (1) culture at 24 or $37^{\circ} \mathrm{C}$; (2) preparation of mega islets exposed to $95 \%$ oxygen in culture; (3) pre-treatment with anti-Ia antibodies; (4) pre-treatment with donor-erythrocytes plus anti-Ia antibodies; (5) pre-treatment with erythrocytes depleted of Ia-bearing cells by ultraviolet light; (6) pretreatment of islets with antibodies against dendritic cells.
In dogs islet culture plus a single injection of anti-lymphocyte serum induced increased survival of allogeneic islets. The yield of islets from human pancreas remains unsatisfactory.

Dr. D. Lorenz (Berlin) described further developments of his techniques for isolation of human islets. Further reports concentrated on the use of cyclosporin A and its immunosuppressive effect in clinical and experimental pancreas transplantation. At present, it is controversial as to whether cyclosporin A influences insulin secretion. Furthermore, several discussants stressed the point that cyclosporin A should not be given together with anti-lymphocytic globulin because of the risk of white cell dyscrasias.

Dr. J. Dupre and Dr. C. R. Stiller (Ontario) reported on their first experience of cyclosporin $A$ in the treatment of 41 patients with recent onset Type 1 (insulin-dependent) diabetes. Patients treated within 6 weeks from diagnosis had better results than those treated 6-44 weeks after diagnosis of diabetes. Of 30 patients in the former group, 16 showed remission, i.e. no concomitant insulin treatment was needed. The cyclosporin A dose was $10 \mathrm{mg} / \mathrm{kg}$ body weight to start with and then serum levels were maintained at $120-130 \mathrm{ng} / \mathrm{ml}$. Some patients showed mild anaemia of unknown origin and $30 \%$ developed gingivae-hyperplasia. Treatment was continued for 1 year.

During the second part of the Workshop, intensified insulin therapy and insulin pumps were discussed. Some groups reported on the application of microcomputers for the calculation of insulin doses. Calculations were performed on the basis of the actual blood glucose concentration, taking glucose control and insulin dosage during preceding days into account. Dr. A.M. Albisser (Toronto) presented a commercially available pocket computer programmed for this purpose.

New insulin pumps were introduced in an exhibition and in communications. These pumps are smaller than previous ones, have greater programming facilities, improved electronics and more alarm functions. In the field of implantable pumps, two new models (Medtronic and Pacesetter) will soon be available for use in human beings. Besides these technical reports there were communications on the stability of pump insulins. It is evident that absolutely stable insulins are not yet available. Moreover, stability depends on the syringe and catheter materials used. Dr. S. G. Melberg (Bagsvaerd, Denmark) reported on changes in insulin solutions after contact with different infusion catheters. Apparently, selection of materials in contact with insulin is not only important in implantable but also in externally worn pumps.

There were several communications on the effects of normalization of blood glucose control on hormone and metabolite concentrations. Although an amelioration of these disturbances was found in most experiments, complete normalization has not been observed under all circumstances. Furthermore, reports of the superiority of portal compared with peripheral insulin delivery were discrepant. The in- 
fluence of tight blood glucose control on the late complications of diabetes was reported by four groups in respect of neuropathy. Improvements in pain, motor nerve conduction velocity and autonomic neuropathy were observed in many patients. Reports of changes in sensory nerve conduction velocity were contradictory. This could be due to methodological problems.

Analysis of the European survey of treatment with external insulin pumps was presented by W. Kerner (UIm). The most notable observation was high incidence of ketoacidosis and severe hypoglycaemia. This was 0.18 and 0.11 per 100 days respectively. It should be noted that the frequency of hypoglycaemic coma exceeded 1 per 100 days in six patients and similarly for 13 patients in respect of ketoacidosis. The mean incidence of subcutaneous infection was reported to be 0.27 per 100 days. Problems related to the technical malfunctions were observed at frequencies of up to 0.35 per 100 days. Dr. H. Kritz (Vienna) gave a report on the international registry of implantable pumps.
A session was dedicated to new developments in the field of implantable glucose sensors. Dr. M. Shichiri (Osaka) presented a sensor working on an enzymatic basis for 3 days in subcutaneous tissue. Dr. U. Fischer (Karlsburg) reported on similar experiments with a sensor implanted into dogs. Tissue and blood glucose concentrations run parallel in these experiments with a time lag of about $15 \mathrm{~min}$.

The next meeting of the Study Group will take place in Igls in February 1985 .

Professor E. F. Pfeiffer

Zentrum für Innere Medizin

Universität Ulm

Steinhövelstraße 9

D-7900 Ulm (Donau)

FRG 